\title{
GLACIER FLOW IN A CURVING CHANNEL
}

\author{
By Keith Echelmeyer
}

(Geophysical Institute, University of Alaska-Fairbanks, Fairbanks, Alaska 99775-0800, U.S.A.)

and BARCLAY KAMB

(Department of Geological and Planetary Sciences, California Institute of Technology,

Pasadena, California 91125, U.S.A.)

ABSTRACT. The flow of a glacier along a channel of constant longitudinal curvature is analyzed using analytical and finite-element methods. Channels of various crosssectional shape are investigated, ranging from a simple rectangular form with zero shear traction along the bed to realistic profiles taken from Blue Glacier, Washington. Terms in the equilibrium and rate-of-deformation equations which are inversely dependent on radius and a body force which varies transversely across the glacier introduce several characteristic features into the stress and velocity fields of the curving glacier. The stress center line is shifted toward the inside of the bend, causing an asymmetric crevasse pattern and non-zero stress magnitude at the surface on the geometric center line of the channel. The stress field is dependent on the stress exponent in the flow law and is non-linear across the surface. The surface-velocity pattern shows a "tilting" of the usual high-order parabolic form, being skewed toward the inside of the bend. There is a shift in the velocity maximum from the deepest part of the channel. All of these curvature-induced features are dependent on the radius of curvature, actual channel geometry, and stress exponent in the flow law. Model results show excellent agreement with the velocity and crevasse patterns on the curving Blue Glacier.

\section{INTRODUCTION}

Glaciers are seldom straight along their entire length. Instead, their channels of ten take a curving path through the mountains, with bends ranging from gentle arcs to abrupt right-angle turns. One might expect that this longitudinal curvature in a glacier valley would influence the distribution of stress and velocity, as is the case in rivers. However, the great difference in Reynolds and Froude numbers between glacier flow and river flow can be expected to cause the stress and flow fields in a curving glacier to be quite different from those in a curving river.

Blue Glacier in the Olympic Mountains of Washington, U.S.A., flows around a bend of nearly constant curvature for much of its length (see Fig. 1). The patterns of crevassing and areal strain-rate, which are not symmetric about the channel center line (as described by Allen and others, 1960; Meier and others, 1974; Echelmeyer, unpublished), have been qualitatively explained by Meier and others (1974) as the result of the longitudinal curvature of the valley. In the present paper we present a quantitative description of the distribution of stress and velocity in a curving glacier, and show that the features observed on Blue Glacier can be explained in detail by taking into consideration this longitudinal curvature. The treatment is an extension to curving channels of the analysis of flow in straight cylindrical channels given by Nye (1965).

\section{GOVERNING EQUATIONS}

Conservation of linear momentum in cylindrical coordinates $(r, \theta, z)$ is expressed by the following set of equations:

$$
\begin{gathered}
\frac{\partial \tau_{r r}}{\partial r}+\frac{1}{r} \frac{\partial \tau_{\theta r}}{\partial \theta}+\frac{\partial \tau_{z r}}{\partial z}+\frac{1}{r}\left(\tau_{r r}-\tau_{\theta \theta}\right)=-f_{r}, \\
\frac{\partial \tau_{r \theta}}{\partial r}+\frac{1}{r} \frac{\partial \tau_{\theta \theta}}{\partial \theta}+\frac{\partial \tau_{z \theta}}{\partial z}+\frac{2 \tau_{r \theta}}{r}=-f_{\theta}, \\
\frac{\partial \tau_{r z}}{\partial r}+\frac{1}{r} \frac{\partial \tau_{\theta z}}{\partial \theta}+\frac{\partial \tau_{z z}}{\partial z}+\frac{1}{r} \tau_{r z}=-f_{z}
\end{gathered}
$$

where we have taken the inertial forces to be negligible and where $\tau$ is the Cauchy stress and $\mathbf{f}$ the body force. The components of the rate-of-deformation tensor, $\dot{\epsilon}$, are given as

$$
\begin{aligned}
\dot{\epsilon}_{r r}=\frac{\partial u_{r}}{\partial r}, \quad \dot{\epsilon}_{\theta \theta} & =\frac{1}{r} \frac{\partial u_{\theta}}{\partial \theta}+\frac{u_{r}}{r}, \quad \dot{\epsilon}_{z z}=\frac{\partial u_{z}}{\partial z}, \\
\dot{\epsilon}_{r \theta}=\frac{1}{2} & {\left[\frac{1}{r} \frac{\partial u_{r}}{\partial \theta}+\frac{\partial u_{\theta}}{\partial r}-\frac{u_{\theta}}{r}\right], } \\
\dot{\epsilon}_{r z} & =\frac{1}{2}\left[\frac{\partial u_{r}}{\partial z}+\frac{\partial u_{z}}{\partial r}\right], \\
\dot{\epsilon}_{\theta z} & =\frac{1}{2}\left[\frac{\partial u_{\theta}}{\partial z}+\frac{1}{r} \frac{\partial u_{z}}{\partial \theta}\right]
\end{aligned}
$$

where $\mathbf{u}=\left(u_{r}, u_{\theta}, u_{z}\right)$ is the velocity.

Several differences exist between Equations (1) and (2) and their Cartesian counterparts. Since the element of arc length along the $\theta$-direction is $r \mathrm{~d} \theta$, the Cartesian operator $\partial / \partial x_{2}$ becomes $\partial / r \partial \theta$. The difference in area between two faces of a small-volume element separated by a radial distance $\mathrm{d} r$, the non-parallelism of the constant-angle faces at $\theta$ and $\mathrm{d} \theta$, and the change in the radial direction at different values of $\theta$ give rise to the additional terms such as $2 \tau_{r \theta} / r$ and $-u_{\theta} / r$.

These additional terms introduced by the cylindrical geometry lead to several interesting features of the stress and velocity fields. A qualitative discussion of some of these features is given in this section, while a more quantitative and complete discussion forms the bulk of the paper.

Consider a curving channel of uniform, bilaterally symmetric cross-section. Equation (2d) shows that, if $u_{r}=$ 0 , then $\dot{\epsilon}_{r \theta}=0$ at some radius other than the location of the velocity maximum, where $\partial u_{\theta} / \partial r=0$. The radius at which $\dot{\epsilon}_{r \theta}$ vanishes is shifted inward, toward smaller $r$. In a Reiner-Rivlin fluid such as ice, which obeys a power-law type constitutive relation, the shear stress $\tau_{r \theta}$ will vanish where $\dot{\epsilon}_{r \theta}$ vanishes. Thus the "stress center line" - the locus 


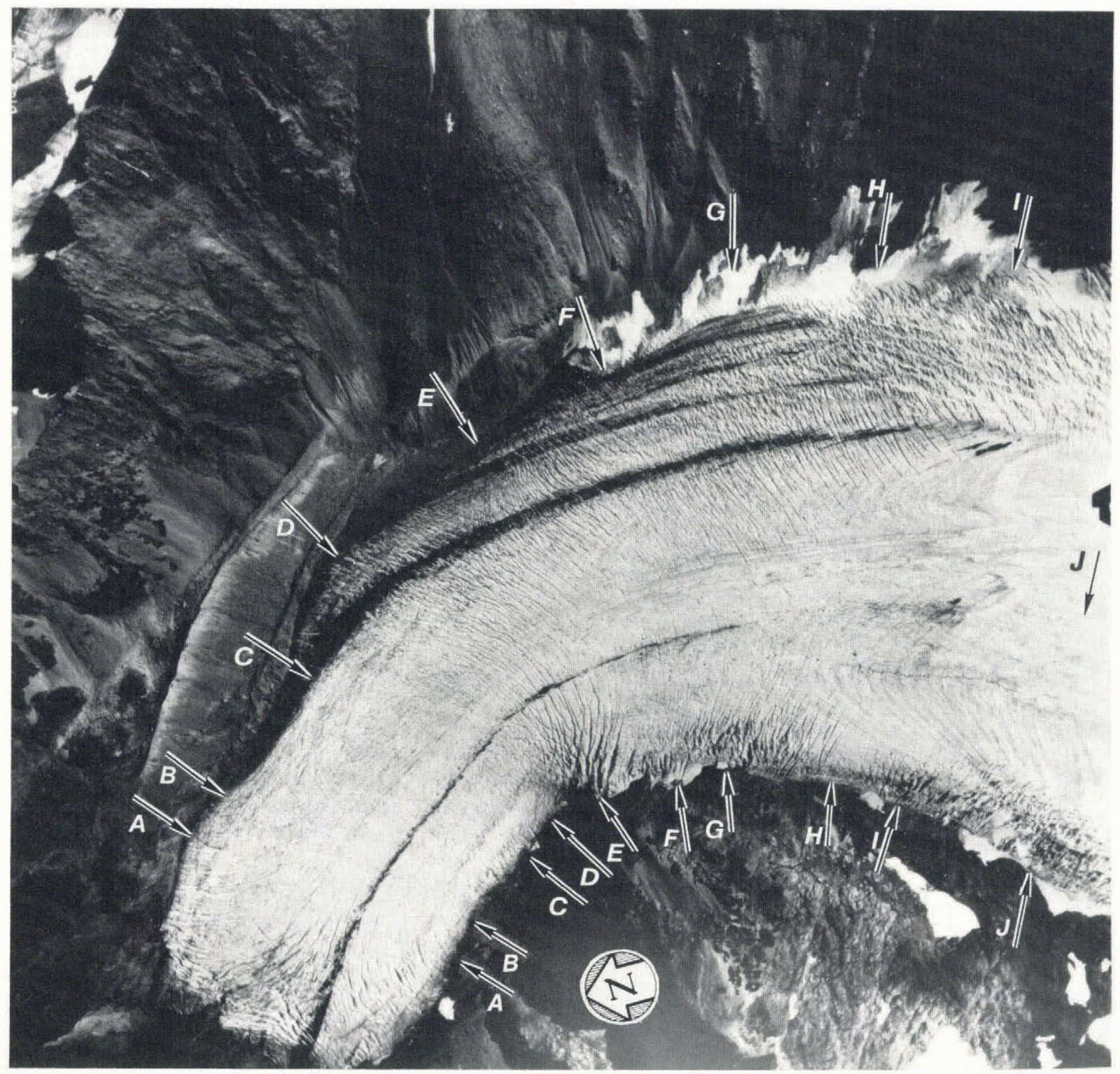

Fig. 1. Vertical aerial photograph of Blue Glacier, Olympic Mountains, Washington, showing curving channel geometry, crevasse patterns, and position of lettered transverse profiles.

of points where $\tau_{r \theta}=0$ (Meier and others, 1974) - is shifted toward a smaller radius than that at which the maximum velocity occurs. Profiles of shear stress and velocity will therefore be asymmetric with respect to the geometric center line of the channel, and the pattern of crevassing will be changed from that in a straight channel, for which the $1 / r$ terms are not present in the Cartesian analogs of Equation (2). These ideas were developed and compared with observations on Blue Glacier by Meier and others (1974). An example of these curvature-induced effects on the surface velocity in a semi-circular channel is shown in Figure 2.

Terms such as $2 \tau_{r \theta} / r$ as in Equation (1b) will give rise to non-linearities in the shear-stress profiles at depth and across the surface of a curving channel which are, again, not present in a non-curving geometry.

The surface of a river in a curving channel often shows a super elevation toward the outside of the bend. This transverse surface slope causes a circulating secondary flow to develop, which is important in the growth of meanders and sediment deposition. In the case of glacier flow, the ratio of centripetal forces to down-stream gravitational forces (a "centripetal" Froude number equal to the transverse slope) is extremely small $\left(\sim 10^{-15}\right)$ and, thus, in the following, we assume that secondary flow is negligible in the motion of a glacier around a bend. A more detailed analysis by Echelmeyer shows that this assumption is strictly valid.

\section{SIMPLE MODEL FLOW IN A RECTANGULAR CHANNEL}

We begin with the discussion of a model that is simplistic and not realistic, but can be treated analytically, and reveals the basic, important features of flow in curving channels.

Consider the flow of ice in a curving, rectangular channel of uniform width, $D$, and depth, with parallel vertical side walls, with no slip on these side walls, and with zero shear traction along the basal interface. The channel center line is a curve with constant radius of curvature, $R_{\mathrm{c}}$, about the $z$-axis, as shown in Figure 3. The channel follows a helical path down the $z$-axis. If $z=$ $Z_{\mathrm{S}}(r, \theta)$ represents the surface of the ice in the channel and $Z_{0}$ is the value of $Z_{s}$ at $\theta=0$, then for a channel of steady, uniform depth and a zero net mass balance

$$
Z_{\mathrm{S}}(r, \theta)=Z_{0}-r \theta \tan \alpha
$$

where $\alpha$ is the surface slope along the channel at radial coordinate $r$. (Ice motion is inclined at an angle $\alpha$ below the horizontal.) 


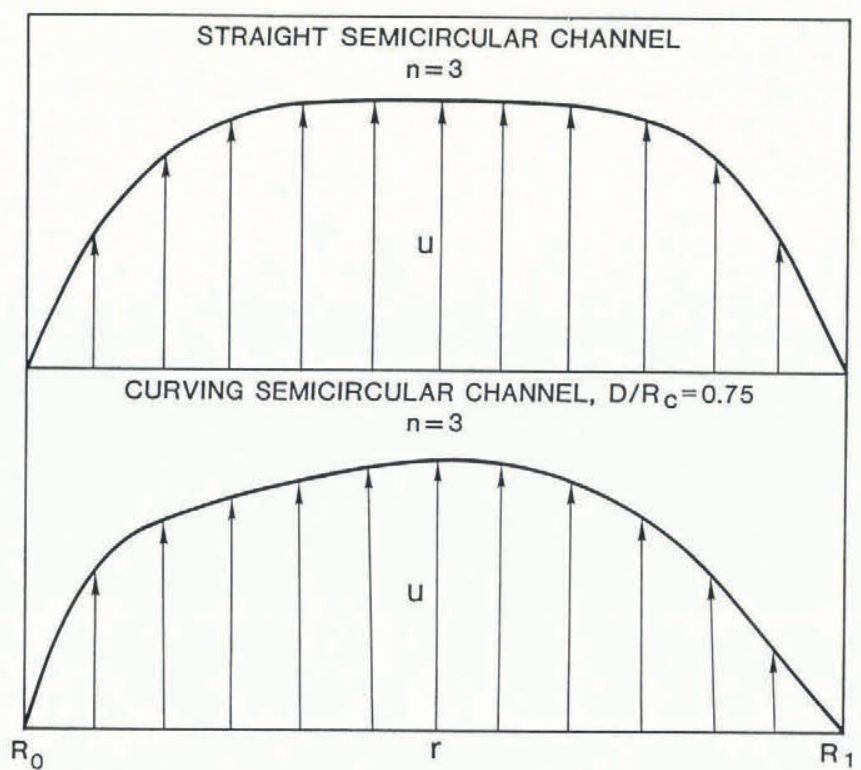

Fig. 2. Example of the influence of curvature on the profile of surface velocity in a semi-circular channel. $D$ is channel width and $R_{c}$ the radius of curvature to the channel center line. $R_{0}$ is the radius to the margin at inside of bend, $R_{1}$ is outer margin.

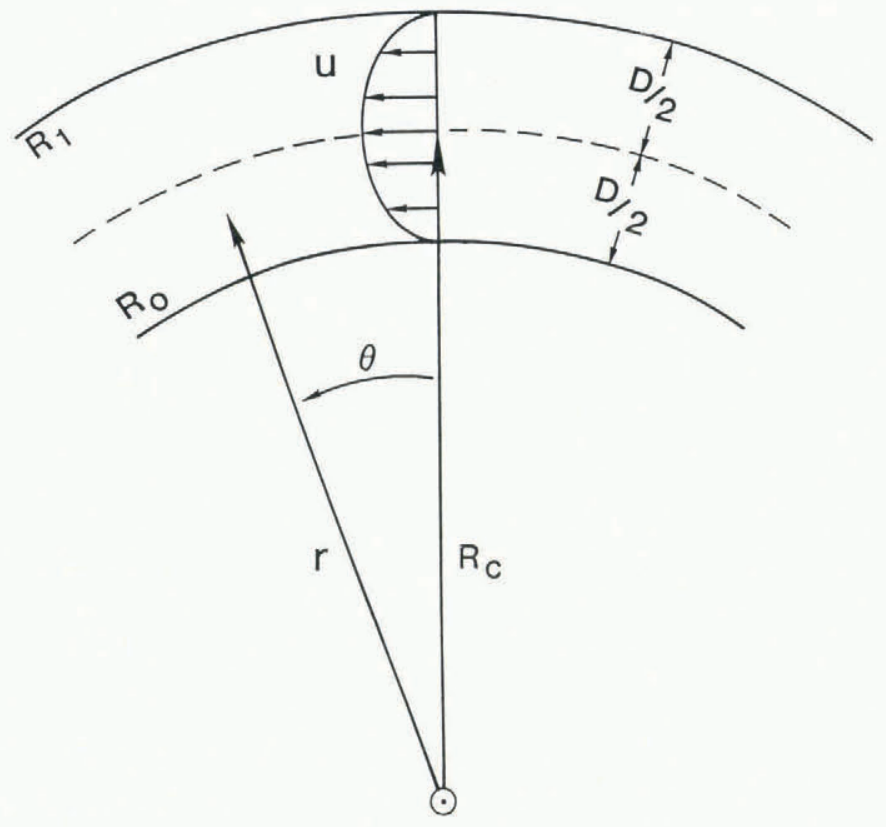

Fig. 3. Plan view of the geometry and notation for the analysis of curving channel flow.

The surface of the ice in a transverse (radial) line across the channel is taken to be level, so that the flow is driven by gravity only in the circumferential $(\theta-)$ direction, and the drop in elevation per unit increment in $\theta$ is taken to be constant, so that the gravitational driving force is independent of $\theta$. Thus the surface slope $\alpha$ must vary inversely with the radial distance $r$ from the center of curvature, over the range from the inside of the bend at $R_{0}$ to the outside at $R_{1}$. Taking $\alpha=\alpha_{0}$ at $R_{0}$, we have

$$
r \Delta \theta \tan \alpha(r)=R_{0} \Delta \theta \tan \alpha_{0}, \quad r \in\left[R_{0}, R_{1}\right]
$$

which, for small surface slopes, can be written

$$
\alpha(r)=\frac{R_{0}}{r} \alpha_{0} .
$$

The variation in surface slope given in Equation (5) is observed on Blue Glacier, as shown in Figure 4. There is a marked decrease in slope from the western (inner) margin to the eastern. The effect is strongest at that part of the channel with the smallest radius of curvature, namely along

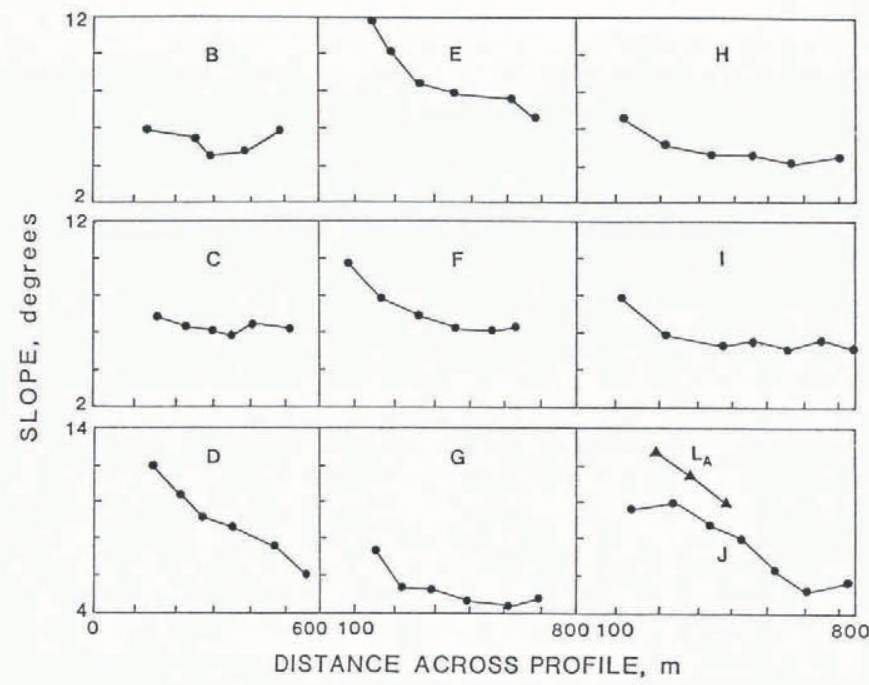

Fig. 4. Observed variation of longitudinal surface slope across transverse profiles on Blue Glacier. Slope was determined over a distance of 2-3 ice thicknesses (from Echelmeyer, unpublished).

profiles D through H. An extreme example is shown along profile $\mathrm{E}$ where a small ice fall exists along the inside of the bend. A similar variation in surface slope is found across the north ice stream of Jakobshavns Isbræ, Greenland, which makes a nearly right-angle bend as it enters the fjord near the grounding zone.

Because the channel has constant cross-sectional shape, and because the effective driving force for flow $(\rho g \alpha)$ is $\theta$-directed and independent of $\theta$, it can be assumed that all of the flow is in the $\theta$ direction, inclined downward to the horizontal at the angle $\alpha$. (This assumption is fully analogous to the assumption that in straight cylindrical channels the flow is everywhere parallel to the cylinder axis (Nye, 1965).) It follows that $u_{r}=0$. The assumption that there is no shear stress across the bottom of the cylindrical channel, as well as across the ice surface, means that there can be no shear of type $\partial u_{\theta} / \partial z$; the flow $u_{\theta}(r)$ is independent of depth $z$ as well as angle $\theta, \tau_{z \theta}$ must therefore be zero (to order $\alpha$ ) and the effective driving force for flow must be supported entirely by the lateral shear stresses of type $\tau_{r \theta}$, which must be independent of $z$.

Taking the $z$-axis vertically upward, the body force can be written $f=(0,0,-\rho g)$. Assuming the hydrostatic condition $\tau_{r r}=\tau_{\theta \theta}=\tau_{z z}$, we have, to order $\alpha, \tau_{z z}=$ $-\rho g\left(Z_{\mathrm{S}}-z\right)$. This then implies that

$$
\tau_{\theta \theta}=-\rho g\left(Z_{0}-r \theta \tan \alpha-z\right)
$$

from Equation (3). (Terms of order $\alpha^{2}$ would enter Equation (6) because the surface has slope $\alpha$ in the $\theta$ direction; these have been neglected.) Substitution of Equation (6) into Equation (1b) and elimination of the term $\partial \tau_{z \theta} / \partial z$ for the reasons given in the previous paragraph yields

$$
\frac{\partial \tau_{r \theta}}{\partial r}+2 \frac{\tau_{r \theta}}{r}+\rho g \tan \alpha=0
$$

or, using Equation (5)

$$
\frac{\partial \tau_{r \theta}}{\partial r}+2 \frac{\tau_{r \theta}}{r}=-\rho g \alpha_{0} \frac{R_{0}}{r} .
$$


Equation (8) can also be obtained in a slightly distorted coordinate frame where the $z$-axis is chosen normal to the glacier surface and $r, \theta$ as above. In this case, the body force $f_{\theta}$ is equal to $\rho g \sin \alpha$, which, in the small angle approximation is equal to $\rho g \alpha_{0} R_{0} / r$. When substituted in Equation (1b) with

$$
\frac{\partial}{\partial \theta}=\frac{\partial}{\partial z}=0
$$

this gives Equation (8). Within this slightly tilted coordinate system, Equation (8) and the relations $u_{r}=u_{z}=0$ hold to order $\alpha^{2}$.

Equation (8) is subject to the boundary conditions of no slip at the vertical walls $r=R_{0}$ and $r=R_{1} . \tau_{r \theta}$ is uniform with depth, as explained above.

Since $u_{r}=0$, Equation (2d) gives

$$
\dot{\epsilon}_{r \theta}=\frac{1}{2}\left[\frac{\partial u}{\partial r}-\frac{u}{r}\right]
$$

where $u$ is written for $u_{\theta}$. In order to complete the specification of the problem, we take a power-law type relation between shear stress and rate of deformation:

$$
\dot{\epsilon}_{r \theta}=A\left|\tau_{r \theta}\right|^{n-1} \tau_{r \theta}
$$

where, again, we have taken $\tau_{r \theta}$ to be the only non-zero deviatoric stress (to second order in $\alpha$ ). The constant $A$ is the standard flow-law parameter (Paterson, 1981, p. 31).

Relations (8), (9), and (10), along with the no-slip boundary conditions, provide a complete set for the solution of shear stress and velocity in the domain $\left(R_{0}, R_{1}\right)$.

Defining non-dimensional variables

$$
\begin{gathered}
\xi=r / R_{0}, \\
\sigma=\tau_{r \theta} / F, \\
U=u /\left[A R_{0} F^{n}\right],
\end{gathered}
$$

and the dimensionless parameter

$$
\xi_{1}=R_{1} / R_{0}
$$

where $F=\rho g \alpha_{0} R_{0}$, leads to the following set of equations:

$$
\begin{gathered}
\frac{\mathrm{d}}{\mathrm{d} \xi}\left(\xi^{2} \sigma\right)=-\xi, \\
\frac{\mathrm{d}}{\mathrm{d} \xi}(U / \xi)=2|\sigma|^{n}(\operatorname{Sgn} \sigma) / \xi,
\end{gathered}
$$

and the conditions that $U(1)=U\left(\xi_{1}\right)=0$.

Solution of Equations $\left(8^{\prime}\right)$ and $\left(10^{\prime}\right)$ gives

$$
\sigma(\xi)=-\frac{1}{2}\left[1-\frac{1}{\xi^{2}}\right]+\frac{\sigma_{0}}{\xi^{2}}
$$

and

$$
U(\xi)=2 \xi \int_{1}^{\xi}|\sigma|^{n}(\operatorname{Sgn} \sigma) \frac{d \xi^{\prime}}{\xi^{\prime}}
$$

where $\sigma_{0} \equiv \sigma(1)$.

The condition of no slip at $\xi=1$ has already been incorporated into Equation (13). Applying the remaining boundary condition at the outer wall leads to an evaluation of the boundary shear stress, $\sigma_{0}$. Once this is obtained, the solution is complete. Equation (13) thus leads to the relation

$$
\begin{gathered}
U\left(\xi_{1}\right)=0=\int_{1}^{\xi_{1}}\left|\frac{1}{\xi^{2}}\left[\frac{1}{2}+\sigma_{0}\right]-\frac{1}{2}\right|^{n} . \\
\operatorname{Sgn}\left[\frac{1}{\xi^{2}}\left[\frac{1}{2}+\sigma_{0}\right]-\frac{1}{2}\right] \frac{\mathrm{d} \xi}{\xi} .
\end{gathered}
$$

For different values of the stress exponent $n$, Equation (14) yields a polynomial of degree $n$ in the unknown $\sigma_{0}$. It is interesting to look at the solution for several values of the stress exponent.

For $n=1$, the solution may be obtained explicitly:

$$
\sigma_{0}=-\frac{1}{2}+\frac{\xi_{1}^{2} \ln \xi_{1}}{\xi_{1}^{2}-1}
$$

and thus

$$
\sigma(\xi)=-\frac{1}{2}+\frac{1 \mathrm{n} \xi_{1}}{\left(\xi_{1}^{2}-1\right)}\left[\frac{\xi_{1}}{\xi}\right]^{2}
$$

and

$$
U(\xi)=-\xi \ln \xi+\xi \ln \xi_{1}\left[\frac{1-\xi^{-2}}{1-\xi_{1}^{-2}}\right] .
$$

Following a similar procedure, solutions for different stress exponents may be obtained. For $n>2$, Equation (14) was solved numerically for $\sigma_{0}$, and Equations (12) and (13) were then used to obtain the shear stress and velocity. This procedure indicates in important feature of the stress field, namely, a dependence of shear stress on the exponent $n$, as is seen in Equations (12) and (14). In addition to this flowlaw dependence, the stress and velocity depend upon the radius of curvature and channel width. Shear stress is a non-linear function of radius. Both shear stress and velocity are asymmetric about the channel center line.

For illustration purposes, let $R_{0}=400 \mathrm{~m}$ and $R_{1}=$ $1300 \mathrm{~m}$ (typical values for Blue Glacier, with $R_{\mathrm{c}}=850 \mathrm{~m}$ and $D=900 \mathrm{~m}$, so that $\left.\xi_{1}=3.25\right)$. The shear stress $\tau_{r \theta}$ and velocity $u$ obtained from Equations (11) and (12)-(14) are to be compared with those found in a straight channel of width $D$ and slope equal to the center-line slope of the curving channel $\alpha_{\mathrm{c}}=\alpha_{0} R_{0} / R_{\mathrm{c}}$ with equivalent boundary conditions (as derived by Nye (1952)). To this end, let the normalized stress and velocity be

$$
T_{r \theta}=\tau_{r \theta} /\left[\frac{1}{2} \rho g \alpha_{c} D\right]
$$

and

$$
v=u /\left[\left(\frac{1}{2} \rho g \alpha_{\mathrm{C}} D\right]^{n}{ }_{A D / 2(n+1)}\right] .
$$

If the radius of the stress center line is $R_{\tau}$ and the radius at which the maximum velocity occurs is $R_{\mathrm{v}}$, then the position of these two radii from the channel center line relative to the channel width, denoted $\rho_{\tau}$ and $\rho_{\mathrm{V}}$, respectively, is given by

$$
\begin{aligned}
& \rho_{\tau} \equiv\left(R_{\tau}-R_{\mathrm{c}}\right) /(D / 2), \\
& \rho_{\mathrm{v}} \equiv\left(R_{\mathrm{v}}-R_{\mathrm{c}}\right) /(D / 2) .
\end{aligned}
$$

For a straight channel with stress center line and velocity maximum at the center of the channel $T_{r \theta}\left(R_{0}\right)=T_{r \theta}\left(R_{1}\right)=$ $\nu_{\max }=1$ and $\rho_{\tau}=\rho_{\mathrm{V}}=0$; if the stress center line is shifted completely to the inner channel wall $\left(R_{0}\right)$, then $\rho_{T}=$ -1 , etc. Table $I$ and Figures 5 and 6 show the results for the curving channel described above, with different values of $n$. Table II shows the variations in $\rho_{\tau}$ and $\rho_{\mathrm{V}}$ with decreasing curvature for both $n=3$ and $n=1$. All results are given as functions of the dimensionless transverse coordinate $r / R_{0}$ and parameter $R_{1} / R_{0}$, which is a measure of the dimensionless relation between center-line curvature $R_{\mathrm{c}}$ 


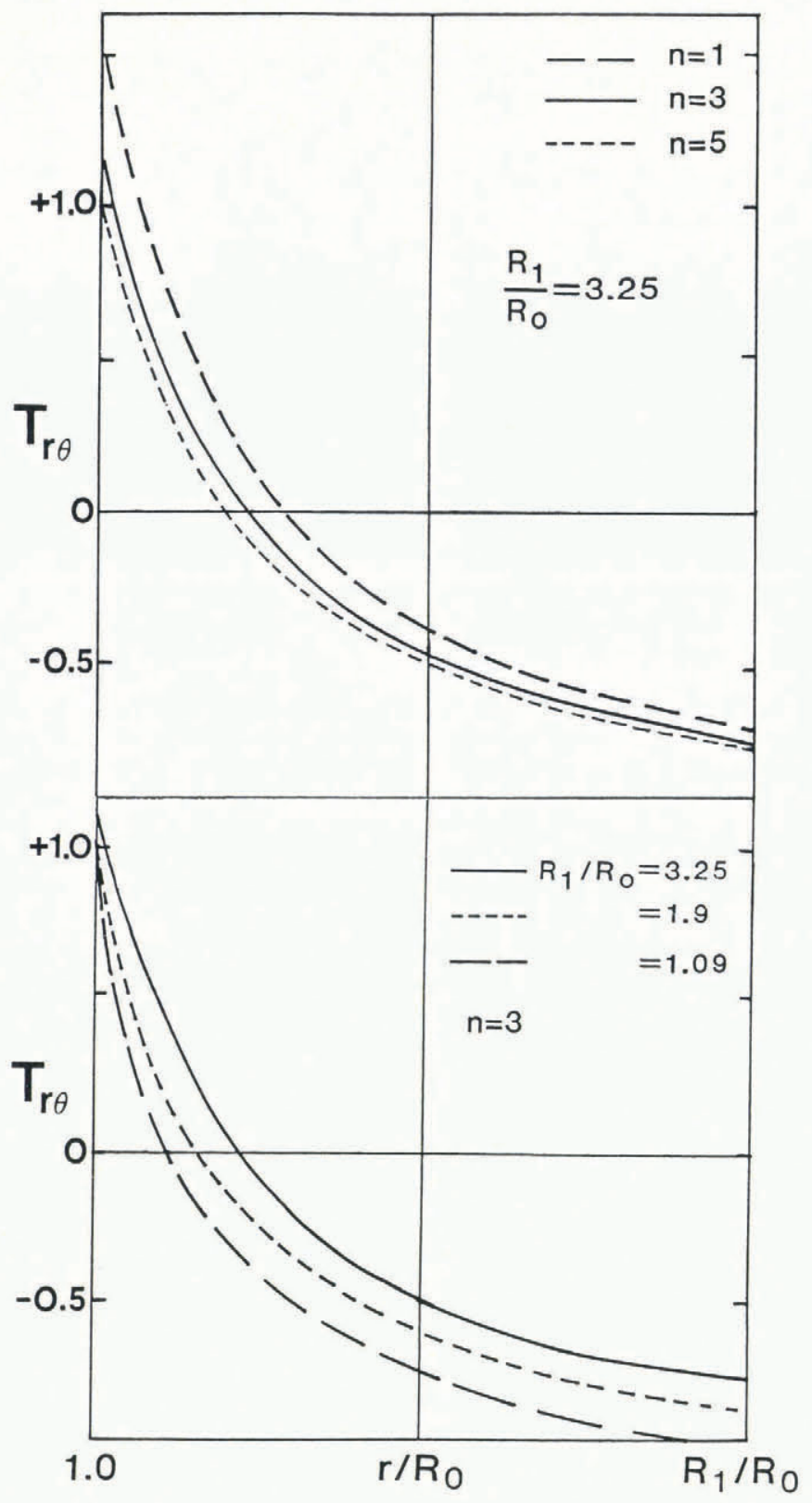

Fig. 5. Normalized shear stress $\left(T_{r \theta}\right)$ across the width of a curving, rectangular channel with zero traction at the bed, for: (a) different stress exponents, and (b) different curvatures. Curves are obtained from the analytical discussion in the text.

and channel width $D$, that is, $D / R_{\mathrm{C}}=2\left(R_{1} / R_{0}-1\right) /$ $\left.R_{1} / R_{0}+1\right)$

The effects of channel curvature are illuminated by the results from this simple model. These effects are as follows:

1. The stress center line is shifted toward the inside of the bend. The amount of this shift increases with the curva-

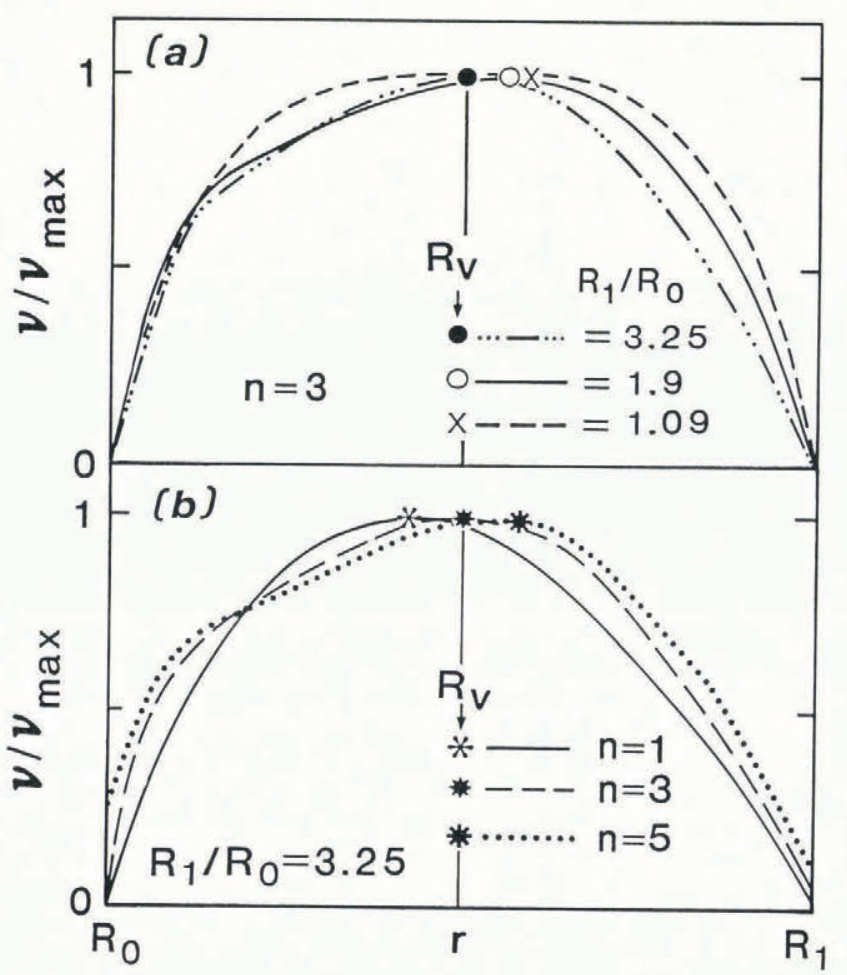

Fig. 6. Velocity profiles across a curving, rectangular channel with zero traction at the bed, for: (a) different curvature with $n=3$; (b) different stress exponents at fixed curvature. Position of maximum velocity is indicated by symbols for the different models.

ture of the channel. For a straight channel, the stress center line is at the channel center, while for tight bends, it tends toward the inside edge.

2. For a fixed curvature, the higher the exponent $n$ the more pronounced the inward shift of the stress center line.

3. The magnitude of $\tau_{r \theta}$ is dependent upon the channel curvature and $n$.

4. $\tau_{r \theta}$, as a function of transverse coordinate $r$, shows an increasing departure from linearity with decrease in $R_{\mathrm{c}}$ (increasing curvature).

5. The velocity profile is no longer symmetric about the center. The asymmetry increases as $R_{\mathrm{c}}$ decreases or $n$ increases.

6. The position of the maximum velocity shifts with changing curvature and stress exponent. For fixed curvature, it moves outward, toward the outside margin, as $n$ increases. For $n<3$, the maximum velocity occurs at a point inside the center, while for $n=3$ the maximum occurs at or outside of the center (depending on $R_{\mathrm{c}}$ ), and, for $n>3$ it is located toward the outside of the

TABLE I. SHEAR STRESS AND VELOCITY IN CURVING CHANNEL COMPARED WITH A STRAIGHT CHANNEL OF EQUIVALENT WIDTH FOR $D / R_{0}=2.25$ $\left(\rho_{\tau}\right.$ AND $\rho_{\mathrm{V}}$ AS DEFINED IN EQUATION (18))

Center line

Shear stress

$\begin{array}{ccc}n & T_{r \cdot \theta}\left(R_{0}\right) & T_{r \theta}\left(R_{1}\right) \\ 1 & 1.52 & -0.71 \\ 3 & 1.16 & -0.75 \\ 5 & 1.04 & -0.76\end{array}$

Stress

$\rho_{\tau}$

$-0.46$

$-0.56$

$-0.60$

\section{Velocity}

\begin{tabular}{rc}
\multicolumn{1}{c}{$\rho_{\mathrm{v}}$} & $\nu_{\max }$ \\
-0.18 & 0.96 \\
0.00 & 0.84 \\
0.12 & 0.70
\end{tabular}




$\begin{array}{llrrrrr} & D / R_{0}= & 2.25 & 0.90 & 0.09 & 0.01 & \rightarrow 0 \\ n=3 & \rho_{\tau} & -0.56 & -0.34 & -0.04 & 0.00 & 0.00 \\ & \rho_{\mathrm{V}} & 0.00 & 0.12 & 0.18 & 0.12 & 0.00 \\ & \nu_{\max } & 0.84 & 0.96 & 0.99 & 1.00 & 1.00 \\ & & & & & & 0.00 \\ & & -0.46 & -0.26 & -0.04 & 0.00 & 0.00 \\ & \rho_{\tau} & -0.18 & -0.10 & -0.02 & 0.00 & 1.00\end{array}$

bend. An interesting feature is noted for $n=3$ and $n=$ 5. As the curvature decreases, there is a shift in the position of the velocity maximum away from the geometric center line toward the outside of the bend. If this trend were to continue, then the position of maximum velocity would not be the channel center line as a straight channel is approached $\left(R_{\mathrm{c}} \rightarrow \infty\right)$, contrary to what is expected from standard flow theory (Nye, 1952). This is explained by the shape of the velocity profile in a curving channel. The profiles in Figure 6 may be approximated by high-order parabolas which are centered at the stress center line and tilted by an angle $\beta$ about this point. The tilt $\beta$ is approximately inversely proportional to $R_{\mathrm{C}}$. The maximum point of the tilted parabola $\left(u_{\text {max }}\right)$ will at first move outward toward a larger value of $R_{\mathrm{y}}$ with decreasing tilt (increasing $R_{\mathrm{c}}$ ); however, as the tilt approaches zero $\left(R_{\mathrm{c}} \rightarrow \infty\right)$, the maximum point is no longer at the apex of the parabola; rather it is on the limb of the parabola facing the inside of the bend. This point of maximum velocity then tends to the channel center line, as already discussed. The value of $\rho_{\mathrm{v}}$ for $D / R_{0}=0.01$ and $n=3$ shows an example of this changing trend. If the parabola is strongly peaked, as for $n=1$, the maximum will not move outward of the center line.

\section{FLOW AND STRESS DISTRIBUTION IN SYMMETRIC CURVING CHANNELS}

The above example gives great insight into the effects of a curving channel on the flow of a glacier. The simple flow geometry, with marginal but no basal drag, is, however, seldom realized in Nature. For this reason, an effort was made to examine the flow of ice in more realistic curving channels, with drag along the entire "wetted" perimeter. An axisymmetric out-of-plane element was added to a finite-element code developed by one of the authors for modeling non-linear visco-elastic deformation (Echelmeyer, unpublished), based on an algorithm by Hughes and Taylor (1978). This quasi-three-dimensional element is termed out-of-plane because flow out of the plane comprising the mesh of the two-dimensional elements is considered, along with the usual in-plane flow. Flow through the cross-section of a curving channel can be modeled, but no gradients are allowed in the out-of-plane direction, here taken to be the $\theta$-direction (i.e. along the length of channel). Thus, the finite-element code is capable of solving the system composed of Equations (1) and (2) for $\mathbf{u}=\left(u_{r}, u_{\theta}, u_{z}\right)$ and all six components of $\tau$ with the only restriction being that $\partial / \partial \theta=0$ in Equations (1) and (2). The in-plane flow $\left(u_{r}, u_{z}\right)$ allows for motion driven by an upper ice surface which is not horizontal in the radial (transverse) direction. Further description of this finiteelement scheme will be found in a forthcoming article (paper in preparation by $\mathrm{K}$. Echelmeyer).

Finite-element solutions were compared with the analytical results obtained above for the simple curving channel geometry (Figs 5 and 6) and with analytical results for the flow of a power-law fluid down an annular conduit and between two rotating co-axial cylinders (Echelmeyer, unpublished). The agreement was excellent in all cases where a sufficiently dense element mesh was used and when the time step was kept small relative to the Maxwell time for the ice.

In all models discussed below a power-law-type constitutive relation and a boundary condition of no sliding along the ice-channel interface was used. A variation in surface slope governed by Equation (5) was employed.

\section{CURVING SEMI-CIRCULAR CHANNEL}

The symmetry of the stress and velocity fields within a straight semi-circular channel is broken by the introduction of channel curvature, as can be seen in the examples shown in Figures 2 and 7 . In a straight channel, contours of shear

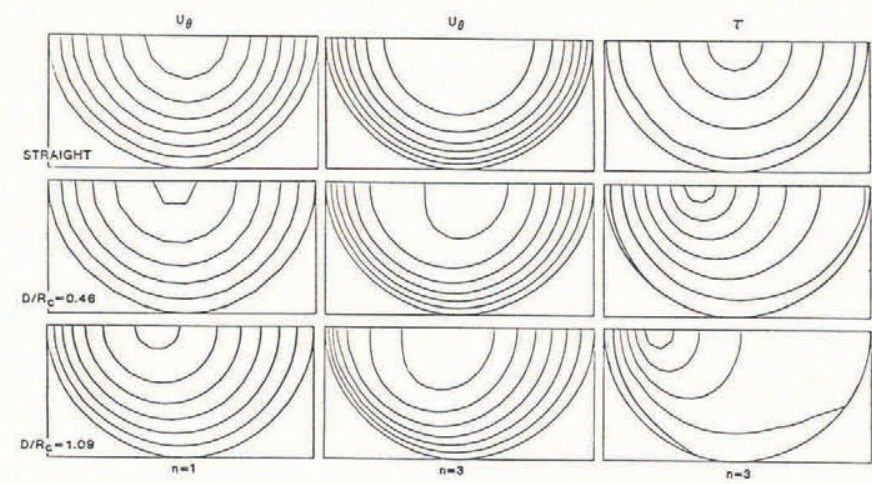

Fig. 7. Contours of velocity and shear-stress magnitude in a semi-circular channel with different curvatures and different stress exponents, from finite-element analysis. Contour intervals are not constant from figure to figure. Diameter of channel is 12 units in Figures 7-10.

stress form concentric semi-circles about the center of the channel, increasing in magnitude outward. Down the central plane and transversely across the surface, the components of shear stress vary linearly. As shown in Figure 7, these patterns change significantly when curvature is introduced. The contours of shear-stress magnitude $\tau$ (where $\left.\tau^{2}=\tau_{r \theta}^{2}+\tau_{z \theta}^{2}\right)$ indicate a substantial shift toward the inside of the bend (left-hand side), which increases with curvature. The highest shear stress and the steepest gradients occur on the inside, and the stress center line $(\tau=0)$ is displaced inward of the geometric center line. For a centerline radius of curvature approximately equal to the diameter of the channel $\left(R_{1} / R_{0}=3\right)$, the stress center line is moved half-way toward the inner margin.

The shear stress $\tau_{r \theta}$ across the surface of the channel (normalized by $\frac{1}{2} \rho g \alpha_{c} D$, where $D$ is the diameter of the channel) is shown in Figure $8 \mathrm{~b}$ for $n=3$. The shift in the stress center line (where $\tau_{r \theta}=0$ ) is apparent, as is the increase in stress at the inside of the bend and a decrease outward of center; this is related to the variation in surface slope across the channel by Equation (5). The stress variation with radial coordinate is non-linear. At depth along the channel center line there is very pronounced non-linear variation in shear-stress magnitude $\tau$, as shown in Figure 

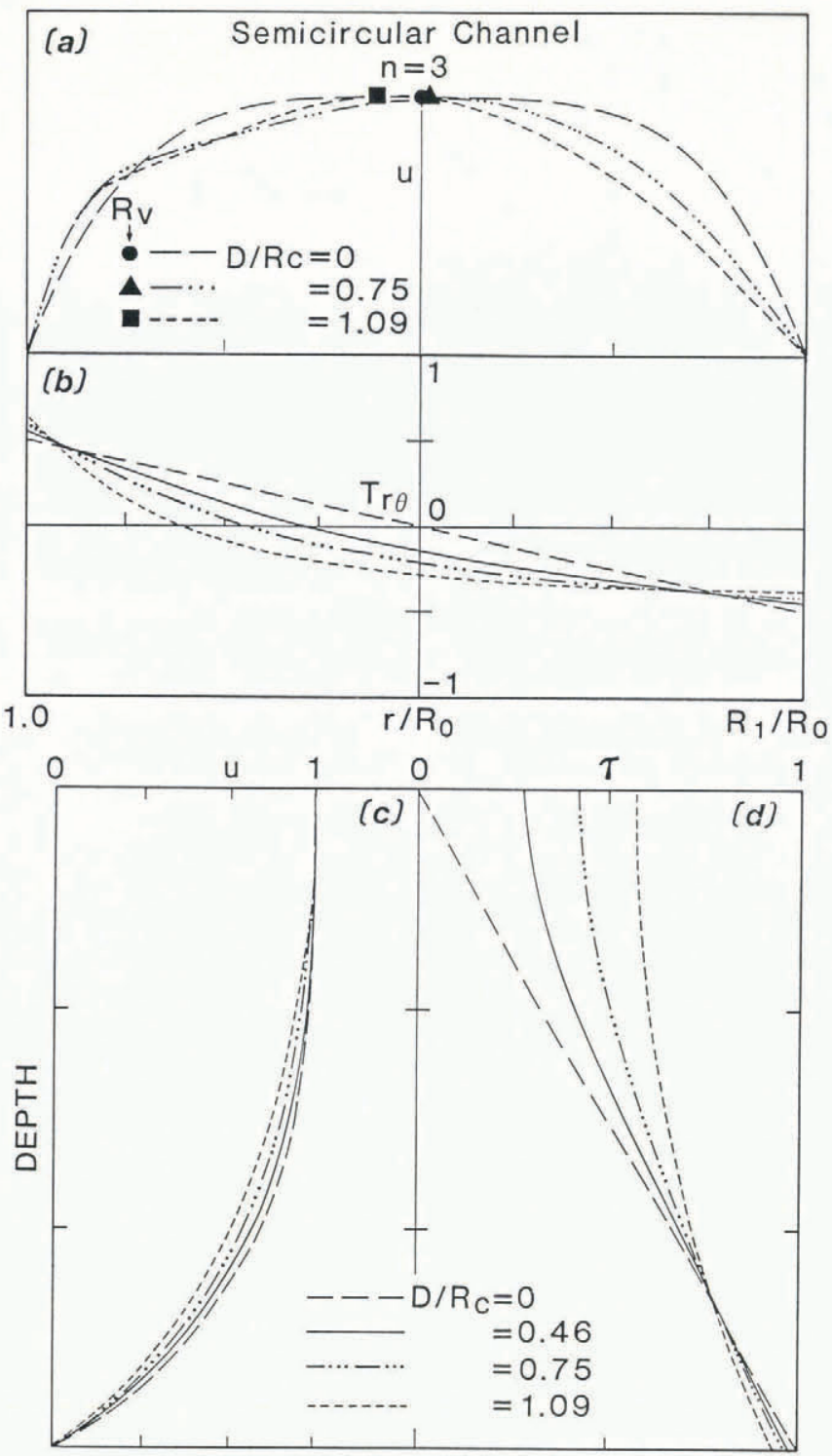

Fig. 8. Effects of increasing channel curvature for semi-circular channel, with $n=3 . \quad$ (a) Surface-velocity profiles and position of velocity maximum, $R_{v}$. (b) Non-dimensionalized shear stress $T_{r \theta}$ across the surface. (c) Velocity profiles down channel center line. (d) Shear-stress magnitude at depth along channel center line. $D / R_{c}=0$ corresponds to a straight channel.

$8 \mathrm{~d}$, whereas there is a linear variation with depth in a straight channel, with $\tau=0$ at the surface. Proper account of this depth variation must be made in any analysis of bore-hole deformation in a channel with longitudinal curvature, for the usual assumptions of constant shape factor and zero surface value will not hold at the channel center line; the failure of these assumptions will strongly influence the effective viscosity values determined from such an experiment. A multiple bore-hole experiment, such as that of Raymond (1973), can take into account the non-linear depth variation of the effective strain-rate $\dot{\epsilon}$ (related to $\tau$ ), but cannot readily be free of the assumption that $\tau_{\theta z}$ varies linearly with depth.

The variation in stress with exponent $n$ is shown in Figure $9 \mathrm{~b}$ and $\mathrm{d}$. The variation is not large, but it is significant in that this (and also Figure 7) is the first indication that within a semi-circular channel the stress distribution can vary with $n$, which complicates the interpretation of bore-hole-tilt experiments. As in the rectangular channels discussed above, the stress center line is shifted inward with increasing $n$.

The velocity profile in Figure 2 clearly shows the asymmetric nature of curving channel flow. The variation of
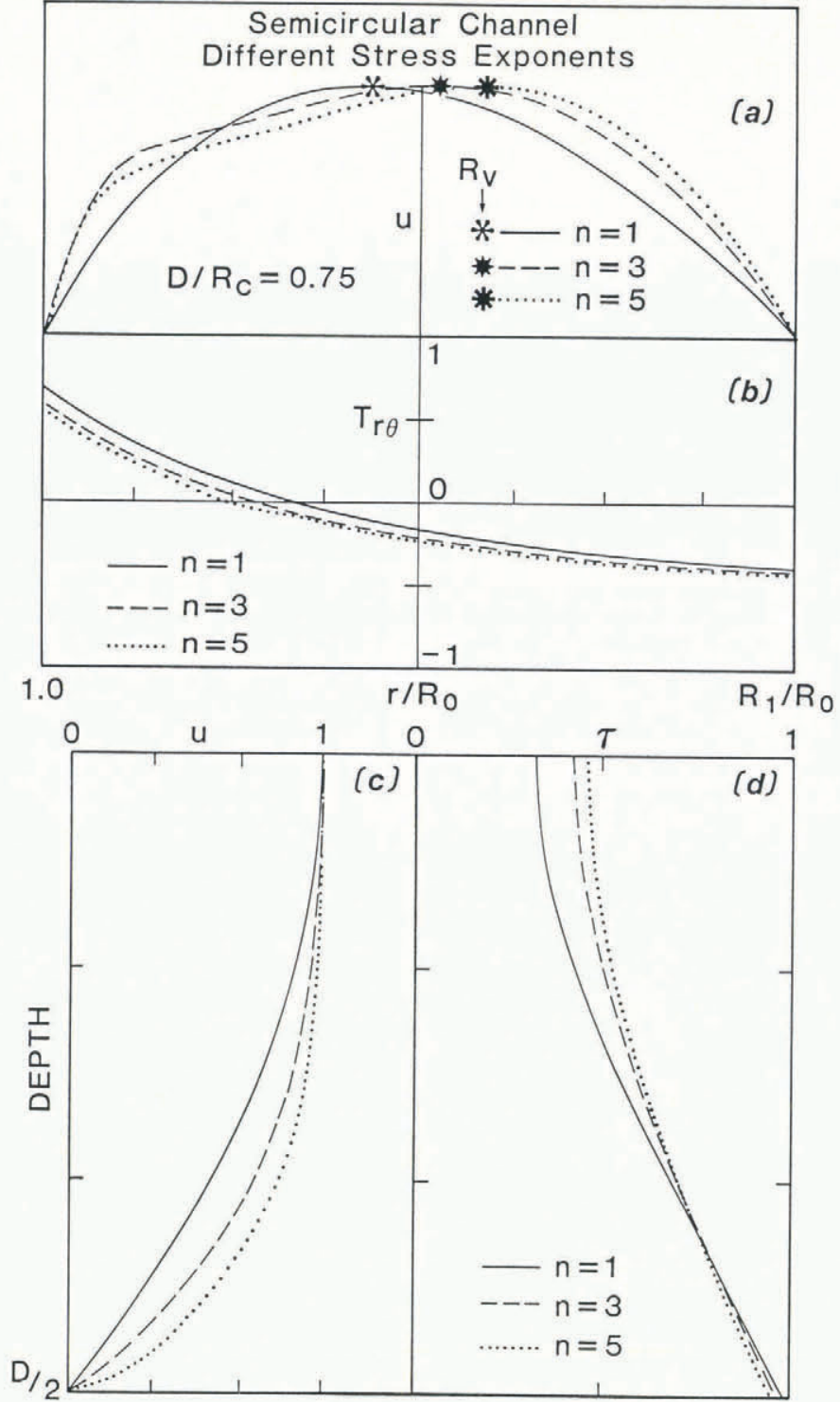

Fig. 9. Effects of a different stress exponent $n$ on velocity and stress in a curving semi-circular channel of fixed curvature with $D / R_{c}=0.75$. (a) Surface-velocity profiles for different $n$ with position of maximum velocity, $R_{v}$. (b) Shear stress across surface. (c) Velocity with depth. (d) Shear-stress magnitude at depth along channel center line. Velocity is normalized by maximum value.

flow velocity $u_{\theta}$ within the channel cross-section for several different values of curvature is shown in Figure 7. As with the stress, the velocity field becomes increasingly asymmetric with increasing channel curvature. Surface-velocity profiles for different radii of curvature at a fixed stress exponent (Figures $8 \mathrm{a}$ and 10a, b) depict several important features of curving channel flow. The profiles show a displacement of the maximum velocity from the center of the channel, steep gradients near the inside of the bend, and gentler gradients toward the outside. And, as found for the rectangular channels, the position of the velocity maximum, $R_{\mathrm{v}}$, can be inside or outside of the geometric center line depending on the value of $R_{\mathrm{C}}$. For fixed $n$, as the curvature decreases the position of the maximum velocity at first moves outward across the channel, but for large $R_{\mathrm{c}}(\rightarrow \infty)$ it moves to the geometric center line. And, as was also found for the rectangular channel above, an increase in the stress exponent at a fixed radius of curvature causes the velocity maximum to be shifted toward the outside of the bend (Fig. 9a).

The magnitude of the maximum velocity in a curving semi-circular channel is very nearly equal to the maximum in a straight channel, within the error of the finite-element models, if the slope of the straight channel is taken to be 


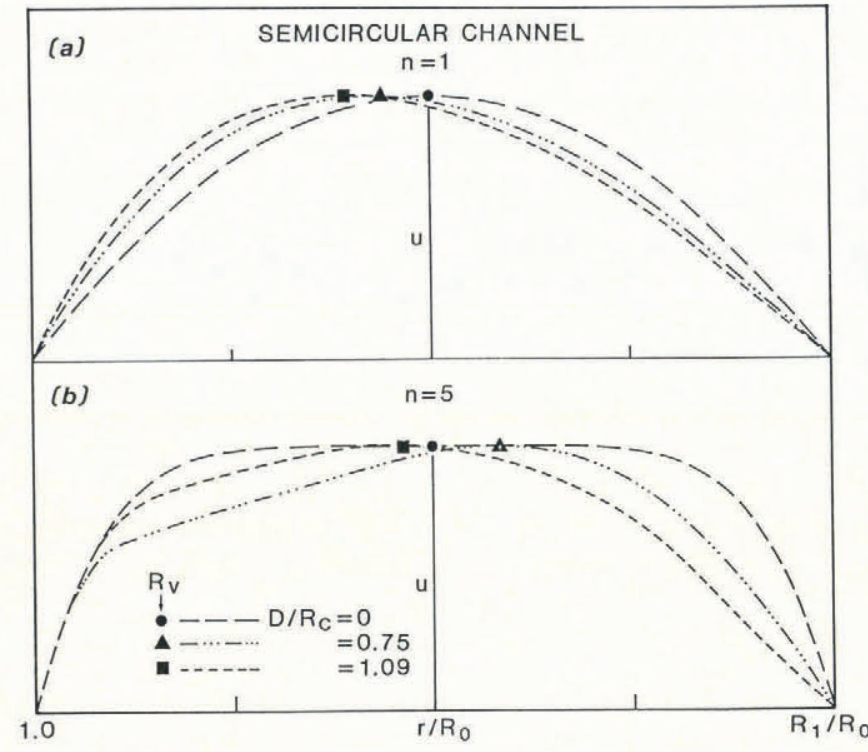

Fig. 10. Profiles of surface velocity in a semi-circular channel with (a) $n=1$, and (b) $n=5$. Position of maximum velocity $\left(R_{v}\right)$ is shown.

equal to the mean value of the surface slope across the curving channel, as obtained from Equation (5) $\left[\bar{\alpha}=\alpha_{0}\left(R_{0} / D\right) \ln \left(1+D / R_{0}\right)\right.$ where $D=$ channel width $]$. Thus, although the distribution of stress and velocity is changed markedly by the introduction of channel curvature, the overall magnitude of the flow is not. (If proper account is taken of the surface-slope variation, this result can be shown to hold for parabolic cross-sections as well.)

Velocity profiles with depth along the channel center line again show a departure from that obtained for a straight channel, as can be seen in Figure $8 \mathrm{c}$ for $n=3$ and different amounts of curvature and Figure $9 \mathrm{c}$ for different stress exponents at fixed curvature. The velocity pattern with depth shows a lower-order parabolic shape with increase in curvature, which, if the channel were assumed to be straight, would mimic an effective stress exponent that is lower than the actual $n$ of the ice.

\section{PARABOLIC CHANNELS}

Nye (1965) and Echelmeyer (unpublished) have shown that there is a non-linear shear-stress distribution with depth along the center line and across the surface of straight parabolic channels. In addition, Echelmeyer (unpublished, paper in preparation) has shown that these stresses depend upon the flow law through the stress exponent $n$. This flow-law dependence of the stresses carries over to channels of realistic, non-symmetric geometry as well.

Channel curvature will tend to introduce asymmetries into these non-linear stress fields, adding another degree of complexity to valley-glacier flow. Figures 11 and 12 show these effects for parabolic channels with various values of curvature and stress exponent. The aspect ratio $W$ (ratio of half-width to depth) of the parabolic channel modeled in these figures was taken to be $W=1.6$, which is a typical value for valley glaciers (Blue Glacier in particular). Other values of $W$ show similar features in their flow and stress fields.

At the channel center line, the shear-stress magnitude (Fig. 11c and d) shows a non-linear variation with depth and a non-zero surface value, similar to that found in the curving semi-circular channel. The non-zero surface value is again due to the shift in the stress center line from the center-line of the channel. The variation in the magnitude of $\tau$ with increase in $n$ from 1 to 5 is on the order of $5-10 \%$.

As with straight parabolic channels, there exists a zone of low shear near the margins. The margin-parallel shear
PARABOLIC CHANNEL, $W=1.6$
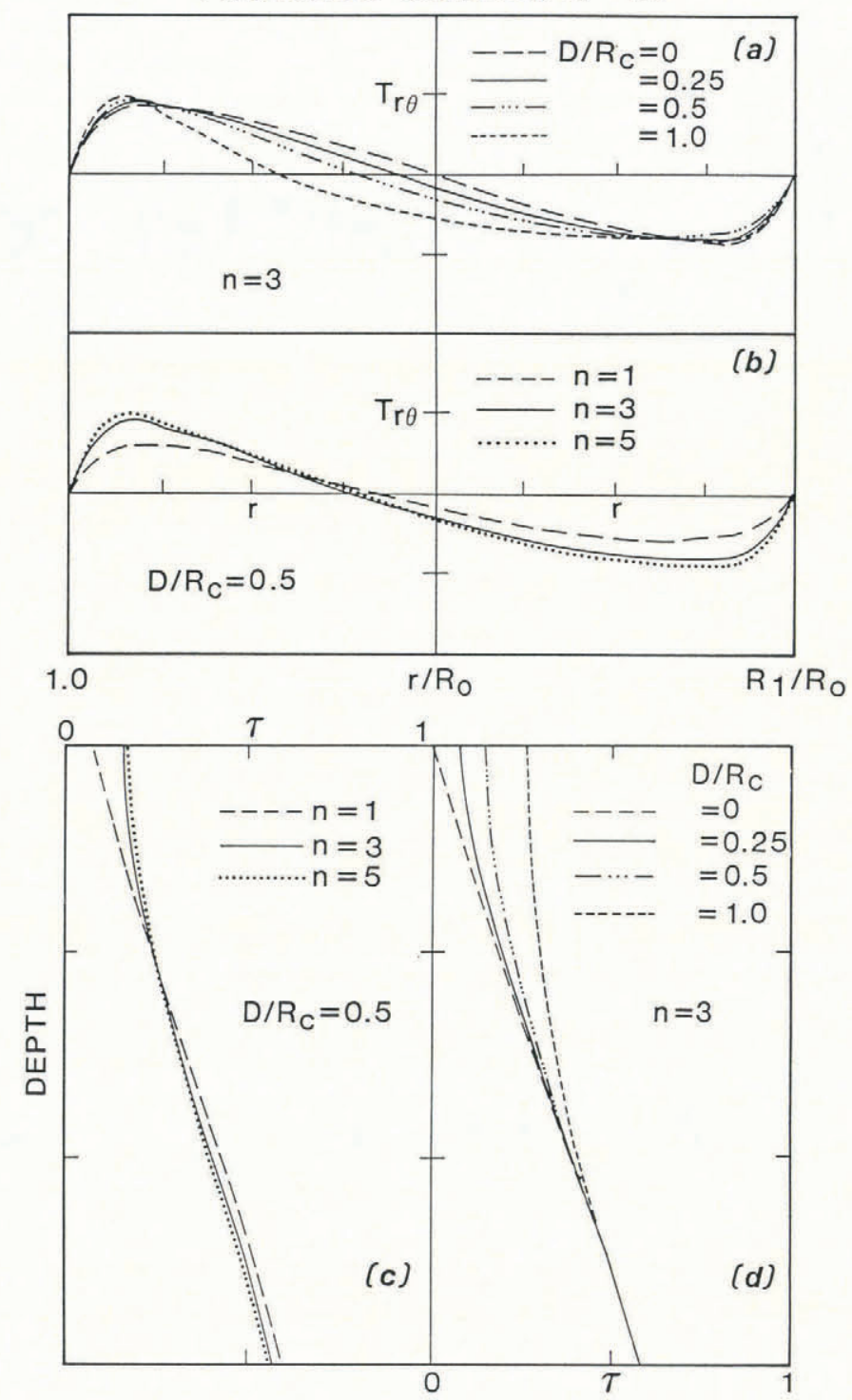

Fig. 11. Surface shear stress $T_{r \theta}$ and shear-stress magnitude $\tau$ down channel center line in a curving parabolic channel, $W=1.6 .(a)$ and (d) show effects of decreasing channel curvature with $n=3$. (b) and (c) show results for different stress exponents $n$ for a fixed curvature $\left(D / R_{c}=0.5\right)$.

stress $\tau_{r \theta}$ at the surface of the channel is shown in Figure $11 \mathrm{a}$ and $\mathrm{b}$. The magnitude and position of the peaks in shear stress vary with curvature and stress exponent. The peak near the inside of the bend moves toward the inside for increasing $n$ and curvature; the peak in $\left|\tau_{r \theta}\right|$ near the outside moves inward with increasing curvature but outward with increasing $n$.

The position of the stress center line is shifted toward the inside of the bend for all curvatures, stress exponents, and aspect ratios $W$, as indicated by the negative values of $\rho_{\mathrm{v}}$ compiled in Figure 13. This is due, as with the rectangular and semi-circular channels already discussed, to the $-u_{\theta} / r$ term in the relation for $\dot{\epsilon}_{r \theta}$, Equation (2d).

As a result of the asymmetries introduced into the stress field by channel curvature, the surface velocity shows a shift of inflection points toward the inside of the bend, with steeper gradients near the inside margin and shallower ones on the outside (Fig. 12). As in semi-circular channels, the position of the maximum velocity can be located inside or outside of the channel center line, depending on the curvature, $n$, and $W$. However, in realistic channels $(W=$ $1-3)$ and with $n=3-5$, only those extremely sharp bends for which $D / R_{\mathrm{C}} \geqslant 1$ have the velocity maximum on the 


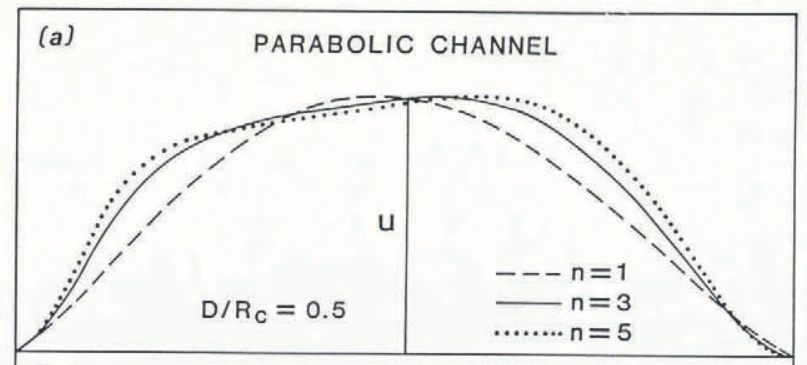

(b)

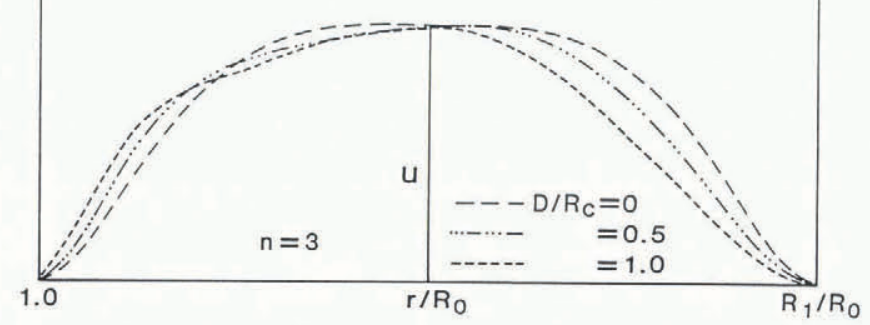

Fig. 12. Surface-velocity profiles for a curving parabolic channel $(W=1.6)$. (a) Profiles for different stress exponents at a fixed radius of curvature $\left(D / R_{c}=0.5\right)$. (b) Profiles for different radii of curvature with $n=3$.
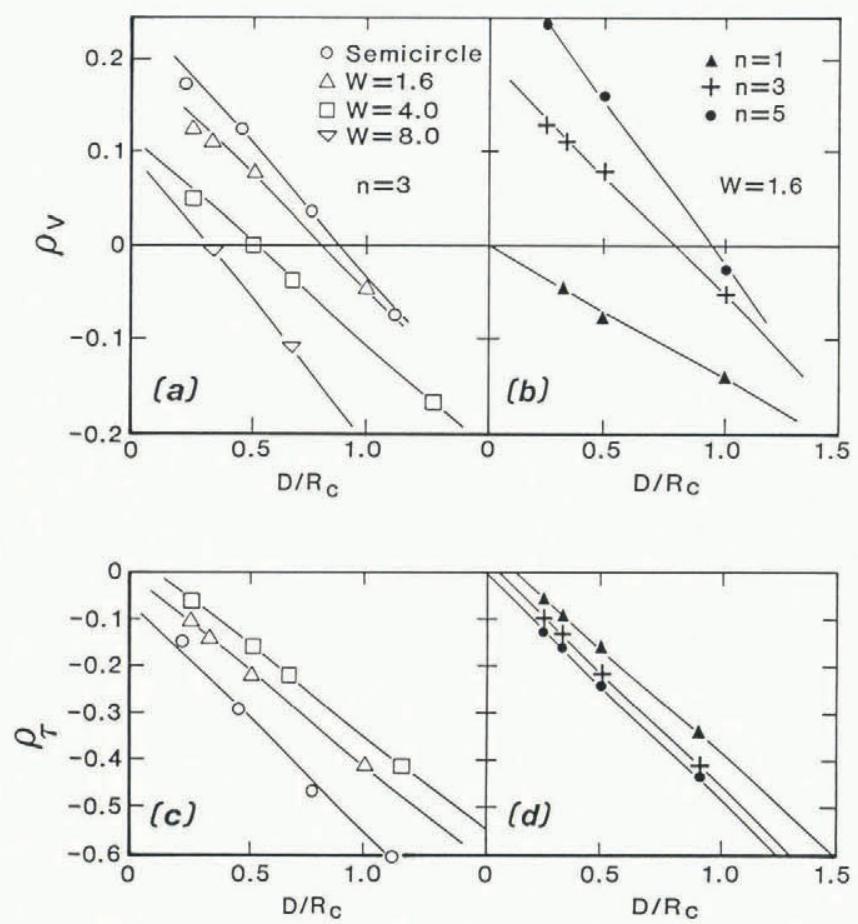

Fig. 13. Position of the velocity maximum, $\rho_{y}$, and the stress center line, $\rho_{\tau}$, as a function of curvature. $D$ is the channel width, $R_{c}$ the radius of curvature to the channel center line, and $\rho_{v}, \rho_{\tau}$ are defined in Equation (18). Symbols represent computed (finite-element) values, lines are interpolated.

(a) $\rho_{v}$ for different channel shapes with $n=3 . W$ is the ratio of half width to depth for parabolic channels.

(b) $\rho_{v}$ for parabolic channel $(W=1.6)$ with different stress exponents.

(c) Stress center line for different channel shapes with $n=3$.

(d) $\rho_{\tau}$ for parabolic channel $(W=1.6)$ with different stress exponents. inside of the channel center line. On the other hand, the velocity maximum for $n=1$ is always toward the inside margin. The velocity profiles (Fig. 12) are very similar in form to those for the rectangular and semi-circular channels already discussed (Figs 2, 6, and 8-10), again showing that curvature will have a marked influence on flow patterns, independent of cross-sectional shape.

As a representative example of curvature-induced flow features, take the cross-sectional shape of Blue Glacier to be approximated by a parabola of $W=1.6$, with a mean depth of $250 \mathrm{~m}$ and width of $800 \mathrm{~m}$. The radius of curvature is approximately $1000 \mathrm{~m}$, so $D / R_{\mathrm{c}}=0.8$. Figure 13 shows that for $n=3-5$, the stress center line should be shifted $120-140 \mathrm{~m}$ inward of the channel center line, while the velocity maximum should be shifted up to $40 \mathrm{~m}$ outward from the center. These shifts agree with those actually observed, although the details of the velocity field do not.

\section{A REALISTIC EXAMPLE: BLUE GLACIER}

The flow of Blue Glacier down its curving channel is strongly influenced by this curvature, as has been shown by Meier and others (1974) and Echelmeyer (unpublished). Qualitatively, the results of the previous sections explain the distribution of the shear component of the rate of deformation, $\dot{\epsilon}_{r \theta}$, at the surface (Meier and others, 1974), the crevasse patterns resulting from the distorted stress field, and asymmetries in the surface-velocity profile. However, the cross-sectional shape of the channel is not truly parabolic or semi-circular, and the channel curvature varies along the glacier. In order to describe this flow in quantitative detail, finite-element models were made of the stress and flow in actual cross-sections as determined by radio echo-sounding (Echelmeyer, unpublished). Examples of this modeling are discussed here, with special emphasis on the effects of channel curvature in non-symmetric channels.

In the models that follow, the surface-slope variation determined by Equation (5) and shown in Figure 3 is used. There is no sliding at the bed. This condition of no sliding agrees with the results of Engelhardt and others (1978),

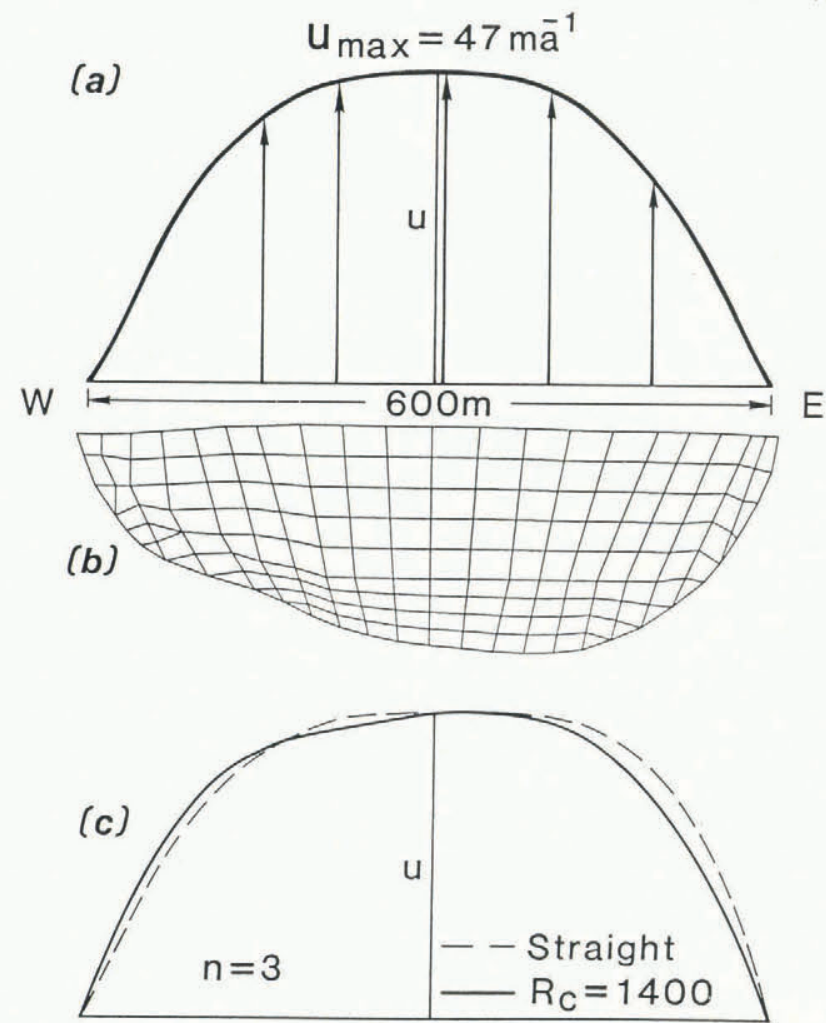

Fig. 14. (a) Observed surface velocity at profile B. (b) Finite-element representation of channel cross-section. Horizontal scale equal to vertical scale. (c) Calculated surface velocity for straight and curving channel. Velocity is normalized to the maximum velocity within the channel, $u_{\max }$ 
Kamb and Echelmeyer (1986), and Echelmeyer (unpublished), which show that, for much of the glacier, basal sliding accounts for only $0-10 \%$ of the total motion. Various stress exponents and radii of curvature were tested for comparison with the observed velocity profiles as measured from August 1977 to August 1978.

Three different profiles are discussed - two (D, F) which are representative of the strongly curved section of the channel (see Fig. 1), and the other (B) across the straighter section of the lower glacier. The finite-element mesh representing the cross-sections, together with the observed profiles of surface velocity, are shown in Figures 14,15 , and 16 .

$$
\mathrm{u}_{\max }=54 \mathrm{ma}^{-1}
$$

(a)

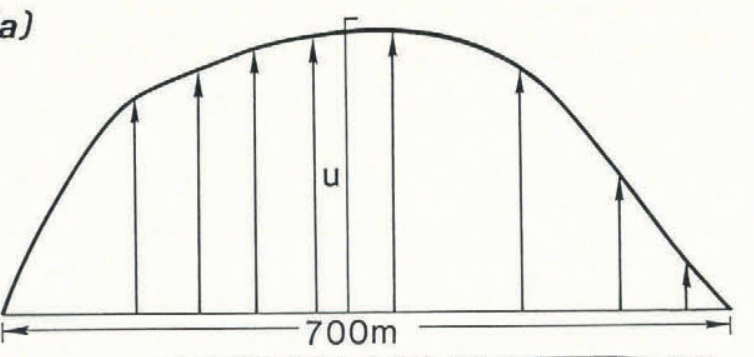

W

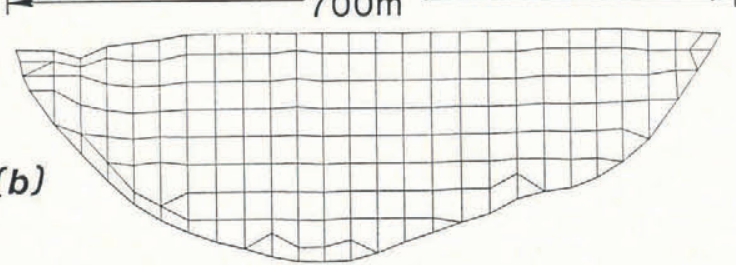

\section{$E$}

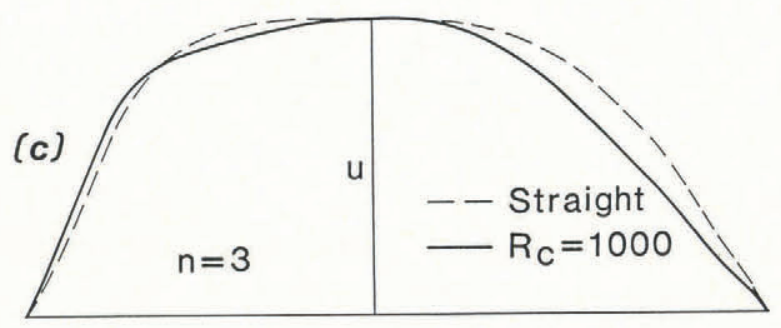

Fig. 15. (a) Observed surface velocity at profile D. (b) Finite-element representation of channel cross-section at $\mathrm{D}$. (c) Calculated surface velocity for straight and curving channel.

The observed velocity profile at $\mathrm{B}$ is nearly symmetric about the channel center line, with a maximum just east of center (right side of Figure 14). The velocity profile obtained from the finite-element model for flow in a straight channel with the cross-sectional shape shown in Figure $14 \mathrm{~b}$ is also nearly symmetric, with only a slight shift in the maximum toward the channel deepening on the east. When a small amount of curvature $\left(R_{\mathrm{c}}=1400 \mathrm{~m}, D / R_{\mathrm{c}}=\right.$ 0.43 ) is introduced, the calculated velocity profile becomes somewhat more asymmetric (Fig. 14c) and the shape departs from that observed. Very little channel curvature at $B$ is apparent in Figure 1 and, thus, the close agreement between a straight-channel model and the observed flow is reasonable. The position of the calculated stress center line agrees well with that inferred from the distribution of $\dot{\epsilon}_{r \theta}$ found by Meier and others (1974) at this location (profile "a" of these authors). The splaying crevasse pattern in this region of the glacier is related to the compression in the ablation zone (Nye, 1952), and is generally symmetric about the geometric center line, as expected from the calculated position of the stress center line.

The flow pattern at profile D (Fig. 15) shows a strong influence of curvature, especially when the channel geometry is considered. The velocity maximum lies $80 \mathrm{~m}$ east of the center line (toward the outside of the bend), while the thickest ice is about $50 \mathrm{~m}$ west of the center line With a radius of curvature of $1000 \mathrm{~m}\left(D / R_{\mathrm{c}}=0.7\right)$ and $n=$

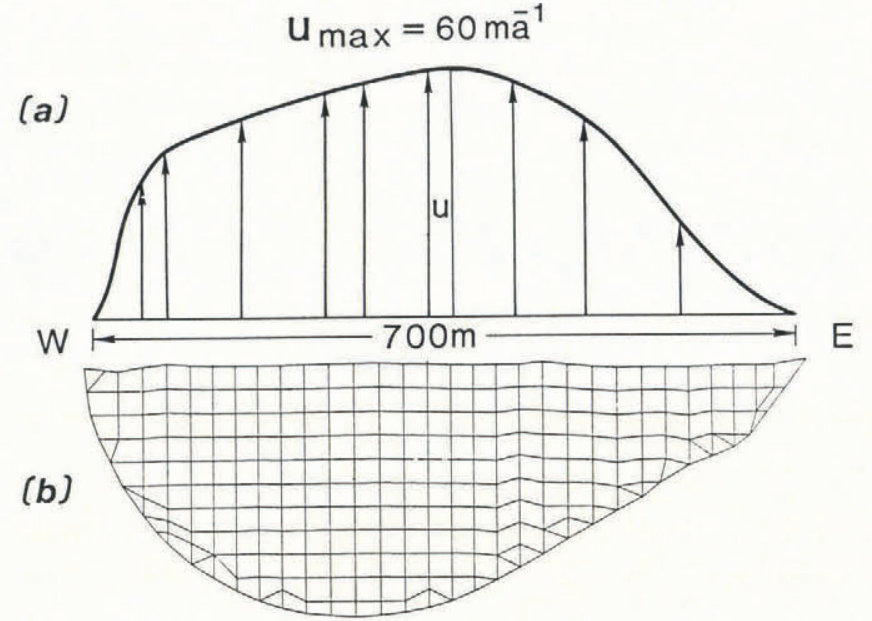

(c)

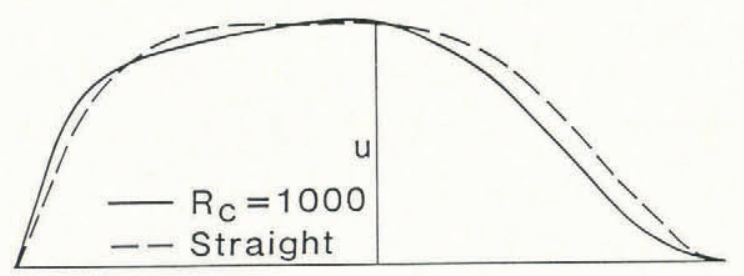

(d)

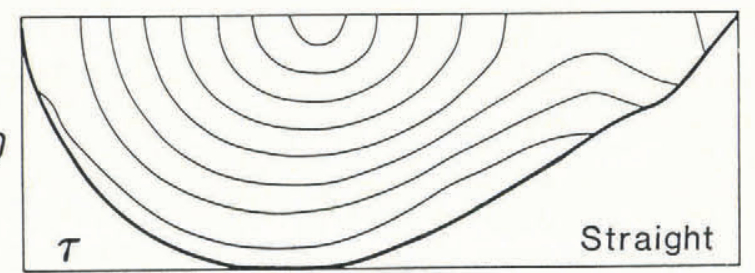

(e)

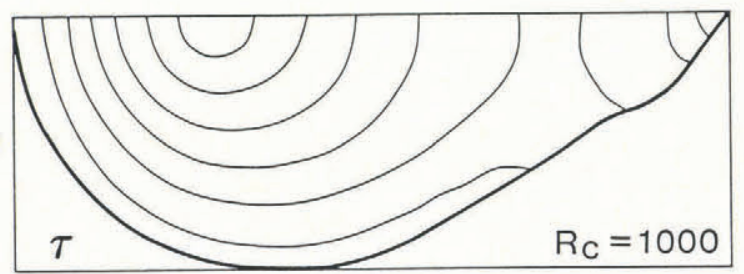

Fig. 16. (a) Observed surface velocity at profile F. (b) Finite-element representation of channel cross-section at $\mathrm{F}$. (c) Calculated surface velocity for straight and curving channel. (d) and (e) Contours of effective shear stress in channel at $\mathrm{F}$ for straight (d) and curving (e) channel $(n=3)$.

$3-4$, the modeled flow agrees with that observed. The stress center line is shifted approximately $120 \mathrm{~m}$ to the west, or more than one-third of the distance to the inner margin. This agrees well with the observed crevasse pattern (Fig. 1).

The cross-sectional channel profile at $F$ is the most asymmetric of the profiles examined in Blue Glacier. The deepest point lies $100 \mathrm{~m}$ west of the channel center line. Surface velocity, on the other hand, shows a maximum at nearly the same distance to the east of center. The flow model for a straight channel of the observed cross-sectional shape shows a maximum velocity west of center, toward the thicker ice (dashed curve in Figure 16c). If channel curvature with radius equal to $1000 \mathrm{~m} \quad\left(D / R_{\mathrm{c}}=0.75\right)$ is introduced, the shape of the calculated velocity profile and the position of the maximum velocity fit the observations quite well, with $n=3$. The shear-stress profile is shifted toward the inside of the bend, with $R_{\tau}$ located $160 \mathrm{~m}$ to the west of center. This is very close to the position of the point where $\dot{\epsilon}_{r \theta}=0$ as observed by Meier and others (1974) and as indicated by the geometry of the crevasse pattern (Fig. 1).

Good agreement of model results with the observed flow and crevasse pattern was found at the other profiles 
indicated in Figure 1, except at the western end of profile $\mathrm{E}$, where there is considerable marginal sliding; since the model omits this sliding, the discrepancy is not unexpected. The radius of channel curvature that gives the best agreement between model and observed profiles varies along the length of the glacier in the way expected from the longitudinal channel curvature indicated by Figure 1. A stress exponent of about 3.5 gives the best overall agreement.

\section{CONCLUSIONS}

Analytical and numerical models show that longitudinal curvature of the channel in which a glacier flows introduces several interesting and characteristic features into the stress and velocity fields. These features were found to be nearly independent of channel cross-sectional shape, at least in general form, and they can be expected to arise whenever there is marginal drag, although the prominence of the features is affected by the amount of slip at the bed.

Curvature-induced effects include the following:

1. The locus of points along the ice surface at which the margin-parallel shear stress vanishes is shifted from the geometric center line of the channel (where it would be in a straight, symmetric channel) toward the inside of the bend. The tighter the bend, the more pronounced the shift. This shift of the stress center line gives rise to two corollary features of curving flow. (i) The geometry of the crevasse pattern is changed from that in straight, symmetric channels. As shown in Figure 17, crevasses opening in response to the tensile stresses associated with marginal shear and propagating inward toward the shifted stress center line will be longer on the outside of the bend than on the inside. If there is longitudinal extension, the transverse crevassing in the central region of the glacier will be oriented normal to the stream lines at a position shifted toward the inside of the bend from the geometric center line. The crevasse pattern of Blue Glacier (Fig. 1) shows these features. (ii) Along the geometric center line, where bore holes are most of ten drilled, the shear-stress magnitude (or effective stress $\tau$ ) will not vanish at the surface, since $\tau_{r \theta}$ is non-zero there. This, coupled with a non-linear depth dependence
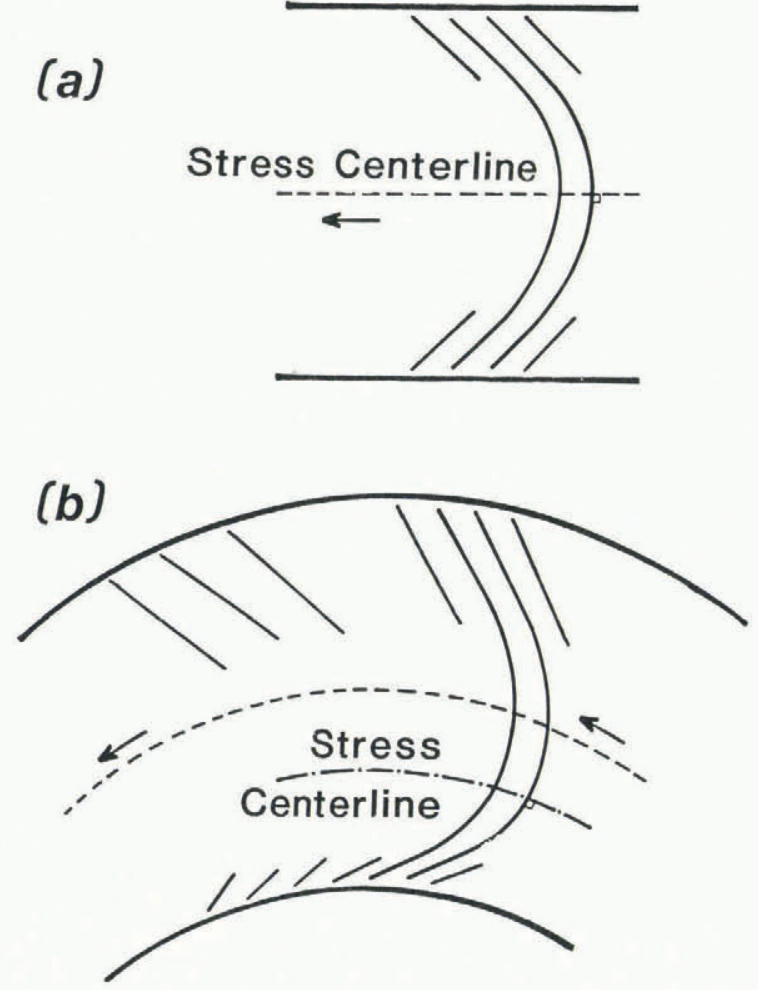

Fig. 17. Crevasse patterns in (a) straight channel and (b) curving channel. When longitudinal extension is large enough, transverse crevasses form perpendicular to the stress center line. of the stress, will make the analysis of bore-hole deformation experiments in terms of a flow relation between stress and deformation rate difficult to perform on curving glaciers.

2. The distribution of stress is dependent on the amount of channel curvature. The resulting dependence of the stress field on the exponent $n$ in the power-law constitutive relation is noteworthy. This dependence was found by Echelmeyer (unpublished) for straight parabolic channels; it is further enhanced by channel curvature. The stress variation can be on the order of $10-15 \%$ for variation of $n$ over a reasonable range of values. This affects the difficulties in interpretation of bore-hole-tilt experiments indicated under 1(ii) above.

3. The profile of flow velocity across the ice surface in a curving symmetric channel is not symmetric. For $n \geqslant 3$, it has the general shape of a higher-order parabola tilted toward the inside of the bend, with a steep gradient $\partial u_{\theta} / \partial r$ near the inside margin of the channel and a gentler gradual gradient toward the outside margin. The stronger the curvature, the more pronounced the tilting. Velocity profiles on Blue Glacier (Figs 14-16) clearly show this feature. As a result of the inward tilting of the profile, the velocity maximum tends to be displaced from the channel center line toward the outside of the bend, and the maximum is sharper than the maximum of the untilted higher-order parabola for a straight channel. For low values of $n(\leqslant 2)$, on the other hand, the tilting is less clearly evident and the velocity maximum is displaced toward the inside of the bend. For typical valleyglacier cross-sections in which the width is about three times the depth, the effects of channel curvature appear generally to have a stronger influence on the position of the velocity maximum than do the details of the crosssectional shape, such as the position of the deepest point.

4. The maximum velocity in a uniformly curving channel is nearly the same as that found in a straight channel of the same cross-section, if the mean effective body force in the curving channel is the same as that in the straight channel.

The foregoing features of curving flow can be well understood in terms of three underlying effects: (i) the $-u_{\theta} / r$ term in the equation for $\dot{\epsilon}_{r \theta}$ in cylindrical polar coordinates; (ii) the $2 \tau_{r \theta} / r$ term in the equation for stress equilibrium in the $\theta$ direction; (iii) the inverse variation of surface slope with radial distance $r$ from the center of curvature for a glacier flowing around a bend of uniform curvature, with constant channel cross-section. It should be understood that the rate-of-deformation and stressdistribution effects are not due to the polar coordinatesystem geometry per se, because the deformation rates and the conditions of stress equilibrium are independent of the coordinate system in which they are calculated. Rather, they arise because in uniformly curving flow within a constant cross-section channel the horizontal flow velocity is directed everywhere in the $\theta$-direction of the polar coordinate system.

\section{ACKNOWLEDGEMENTS}

We thank the National Park Service, Olympic National Park, for permission to carry out the field study that acquired the Blue Glacier flow data reported here. The work was done under the support of National Science Foundation grants EAR $78-01715$ and EAR $80-08319$.

\section{REFERENCES}

Allen, C.R., and others. 1960. Structure of the lower Blue Glacier, Washington, by C.R. Allen, W.B. Kamb, M.F. Meier, and R.P. Sharp. Journal of Geology, Vol. 68, No. 6, p. 601-25.

Echelmeyer, K. Unpublished. Response of Blue Glacier to a perturbation in ice thickness - theory and observation. [Ph.D. thesis, California Institute of Technology, 1983.] 
Engelhardt, H.F., and others. 1978. Basal sliding and conditions at the glacier bed as revealed by bore-hole photography, by H.F. Engelhardt, W.D. Harrison, and B. Kamb. Journal of Glaciology, Vol. 20, No. 84, p. 469-508.

Hughes, T.J.R., and Taylor, R.L. 1978. Unconditionally stable algorithms for quasi-static elasto/visco-plastic finite element analysis. Computers and Structures, Vol. 8, p. 169-73.

Kamb, B., and Echelmeyer, K.A. 1986. Stress-gradient coupling in glacier flow: I. Longitudinal averaging of the influence of ice thickness and surface slope. Journal of Glaciology, Vol. 32, No. 111, p. 267-84.
Meier, M.F., and others. 1974. Flow of Blue Glacier, Olympic Mountains, Washington, U.S.A., by M.F. Meier, W.B. Kamb, C.R. Allen, and R.P. Sharp. Journal of Glaciology, Vol. 13, No. 68, p. 187-212.

Nye, J.F. 1952. The mechanics of glacier flow. Journal of Glaciology, Vol. 2, No. 12, p. 82-93.

Nye, J.F. 1965. The flow of a glacier in a channel of rectangular, elliptic or parabolic cross-section. Journal of Glaciology, Vol. 5, No. 41, p. 661-90.

Paterson, W.S.B. 1981. The physics of glaciers, Second edition. Oxford, etc., Pergamon Press. (Pergamon International Library.)

Raymond, C.F. 1973. Inversion of flow measurements for stress and rheological parameters in a valley glacier. Journal of Glaciology, Vol. 12, No. 64, p. 19-44. 